\title{
Medical Image Registration Using a New Information Discrepancy Measure
}

\author{
Shaoyan Sun ${ }^{1, *}$ and Fengnan Sun ${ }^{2}$ \\ ${ }^{1}$ School of Mathematics and Statistics Science, Ludong University, Yantai, China \\ ${ }^{2}$ Clinical Laboratory, Yantaishan Hospital, Yantai, China \\ ${ }^{1}$ sunsy_2014@163.com, ${ }^{2}$ renasun@gmail.com
}

\begin{abstract}
Function of Degree of Disagreement (FDOD), a new measure of information discrepancy, was proposed originally to quantify the discrepancy of multiple sequences. On the one hand, this function has been successfully used in many other fields recently. On the other hand, the Kullback-Leibler divergence (KLD)measure has made great success in multimodality image registration. Comparing these two measures, we find that the FDOD has some peculiar mathematical properties superior to the KLD measure. Motivated by these facts, in this contribution, we introduce the FDOD function to solve the (3-D) multimodality medical image registration problem. Furthermore, we propose a normalized version of the FDOD function which will be more suitable to image registration. Finally, we carried out many experiments to validate our methods. Our results illustrate that the proposed registration methods based on the FDOD function and the normalized FDOD function are feasible and competitive, and compared with the methods based on mutual information and normalized mutual information, the proposed normalized FDOD function performs best in most cases, obtaining subvoxel registration accuracy with higher speed and higher success rate.
\end{abstract}

Keywords: multimodality image registration, kullback-leilber divergence, function of degree of disagreement, mutual information, optimization

\section{Introduction}

The geometric alignment or registration of medical images is a fundamental task in numerous applications in three-dimensional (3-D) medical image processing [1-3]. The key step of image registration is to find a spatial transformation such that a similarity function between two or more images taken at different times from different sensors achieves its maximum. Recently there has been active research into the use of voxelsimilarity-based (VSB) measures of multimodality medical image registration [4-10]. The main advantage of VSB methods is that feature calculation is straightforward or even absent when only grey-values are used, such that the accuracy of these methods is not limited by segmentation errors as in surface based methods. Among these methods, the mutual information (MI) based method is one of the most accurate methods, which measures the statistical dependence between images by measuring the discrepancy between the joint probability distribution and the product of the marginal distributions associated to the case of complete independence by means of the Kullback-Leibler divergence (KLD) measure. Studholme et al improved the robustness of the mutual information by using a normalized mutual information [5]. However, considering the registration accuracy and registration efficiency, there is still a big gap to bring the registration techniques to clinical application.

Received (September 22, 2016), Review Result (January 17, 2017), Accepted (February 2, 2017)

${ }^{*}$ Corresponding Author 
To increase the registration accuracy and efficiency, we introduce the Function of Degree of Disagreement (FDOD), a new measure of information discrepancy, to 3dimensional (3-D) medical image registration. This function is defined originally as a discrepancy measure among multiple sequences. The FDOD function has a close connection with the KLD measure, and in contrast to the KLD measure, it has some peculiar properties, such as symmetry, boundedness, monotonicity, effectiveness in singular case, convexity [11-13]. It has been successfully used in many fields, for example, measuring the discrepancy between DNA sequences, predicting protein structural classes $[14,15]$. All of these facts motivate us to investigate whether this function can be successfully used in the field of medical image registration.

In this paper, we apply this function to evaluate the similarity between two images. Like the method based on the KLD, the FDOD function based method evaluates the similarity between two images by measuring the discrepancy between the joint probability distribution and the product of the marginal distributions associated to the case of complete independence. More importantly, we propose a normalized version of the FDOD function, NFDOD, and apply it to the registration problem too. The purpose to find the optimal registration can be achieved by maximizing the FDOD function or the NFDOD function respectively. In the next section, we first give a description of the FDOD function and define its normalized version, the NFDOD function, and then introduce the use of these two functions to solve the 3-D medical image registration problem. Numerical experiments are demonstrated in Section 3. Finally, in Section 4, we present some concluding remarks.

\section{Medical Image Registration Based on FDOD Function}

\subsection{FDOD function and NFDOD function}

Compared with mutual information, the bounded FDOD function is a relatively new measure that was introduced in the study of information discrepancy among some multiple information sources [11-13]. In the case of measuring the discrepancy of a group of distributions, it is reduced to

$$
\operatorname{FDOD}\left(U_{1}, U_{2}, \cdots, U_{s}\right)=\sum_{k=1}^{s} \sum_{i=1}^{t} p_{i k} \log \frac{p_{i k}}{\sum_{k=1}^{s} p_{i k} / s}, \quad U_{k} \in \Gamma_{t}
$$

Where $\quad \Gamma_{t}=\left\{\left(p_{1}, p_{2}, \cdots, p_{t}\right)^{T} \mid \sum_{i=1}^{t} p_{i}=1, p_{i} \geq 0, i=1,2, \cdots, t\right\} \quad$ denotes distribution, and $0 \cdot \log 0=0,0 \cdot \log 0 / 0=0$ are defined.

To make it relevant to the image registration context, in the following we only consider two distributions $P=\left(p_{1}, p_{2}, \cdots, p_{t}\right)^{T}$ and $Q=\left(q_{1}, q_{2}, \cdots, q_{t}\right)^{T}$, where $\sum_{i=1}^{t} p_{i}=1$, and $\sum_{i=1}^{t} q_{i}=1$. The discrepancy of two such distributions can be measured by the FDOD function as follows:

$$
F D O D(P, Q)=\sum_{i=1}^{t} p_{i} \log \frac{p_{i}}{\left(p_{i}+q_{i}\right) / 2}+\sum_{i=1}^{t} q_{i} \log \frac{q_{i}}{\left(p_{i}+q_{i}\right) / 2}
$$

It can also be measured by the KLD measure, i.e.

$$
K L D(P, Q)=\sum_{i=1}^{t} p_{i} \log \frac{p_{i}}{q_{i}}
$$

Obviously, the FDOD function is symmetric about the two distributions $P$ and $Q$, while the KLD measure is not. So the FDOD function can be viewed as a symmetric version of the KLD measure. More comparisons of the FDOD function and the KLD 
measure can be found in Table 1. From Table 1 it is clear that the FDOD function has more good mathematical properties than the KLD measure.

The FDOD function can also be rewritten in the following manner:

$$
F D O D(P, Q)=-H(P)-H(Q)+2 H\left(\frac{P+Q}{2}\right)
$$

in which $H$ represents the Shannon entropy.

Furthermore, we define the normalized version of the FDOD function (denoted as NFDOD), its expression is as follows:

$$
\operatorname{NFDOD}(P, Q)=-\frac{H(P)+H(Q)}{2 H\left(\frac{P+Q}{2}\right)}
$$

It is not difficult to deduce that the NFDOD function also has boundness, and its value varies from -1 to 0 .

Table 1. Simple Comparison between KLD Measure and FDOD Function

\begin{tabular}{|l|l|l|}
\hline Basic properties & KLD measure & FDOD function \\
\hline Data amout & 2 & $s \geq 2$ \\
\hline Non-negativity & Yes & Yes \\
\hline Identity & Yes & Yes \\
\hline Symmetry & No & Yes \\
\hline Boundedness & No & Yes \\
\hline Absolute continuity & No & Yes \\
\hline Maximum & $\infty$ & $s \cdot \log s$ \\
\hline Monotonicity & No & Yes \\
\hline
\end{tabular}

\subsection{Registration Process}

In this subsection, we apply both the FDOD function and the normalized FDOD function to 3-D medical image registration. Considering the image intensity values, $a$ and $b$, of a pair of corresponding voxels in the two images that are to be registered to be random variables $A$ and $B$, respectively, estimations for the joint distribution $p_{A B}(a, b)$ can be approximated by either Parzen windowing or histogramming [6]. Histogramming is employed in this paper because the approach is computationally efficient. The joint distribution $p_{A B}(a, b)$ denotes that a voxel in image $A$ has intensity $a$ while the corresponding voxel in image $B$ has intensity $b$. Their marginal distributions $p_{A}(a)$ and $p_{B}(b)$ can be respectively obtained as follows:

$$
\begin{array}{r}
p_{A}(a)=\sum_{b} p_{A B}(a, b) \\
p_{B}(b)=\sum_{a} p_{A B}(a, b)
\end{array}
$$

Variables $A$ and $B$ are statistically independent if $p_{A B}(a, b)=p_{A}(a) \cdot p_{B}(b)$, while they are maximally dependent if they are related by a one-to-one transformation $T: p_{A}(a) \cdot p_{B}(T(a))=p_{A B}(a, T(a))$. Two images of the same subject would be geometrically aligned if the discrepancy between the joint distribution $p_{A B}(a, b)$ and the distribution associated to the case of complete independence $p_{A}(a) \cdot p_{B}(b)$ is maximal. In the present paper, we propose to measure this discrepancy using the FDOD function and the NFDOD function respectively. Their expressions are as follows: 


$$
\begin{gathered}
F D O D=\sum_{a, b}\left(p_{A B}(a, b) \log \frac{p_{A B}(a, b)}{\left(p_{A B}(a, b)+p_{A}(a) \cdot p_{B}(b)\right) / 2}\right. \\
\left.p_{A}(a) \cdot p_{B}(b) \log \frac{p_{A}(a) \cdot p_{B}(b)}{\left(p_{A B}(a, b)+p_{A}(a) \cdot p_{B}(b)\right) / 2}\right) \\
N F D O D=-\sum_{a, b}\left(\frac{p_{A B}(a, b) \log p_{A B}(a, b)+p_{A}(a) \cdot p_{B}(b) \log \left(p_{A}(a) \cdot p_{B}(b)\right)}{\left(p_{A B}(a, b)+p_{A}(a) \cdot p_{B}(b)\right) \log \left(\left(p_{A B}(a, b)+p_{A}(a) \cdot p_{B}(b)\right) / 2\right)}\right.
\end{gathered}
$$

Mutual information (MI) and normalized mutual information (NMI) measure the aforementioned discrepancy respectively in the following manners:

$$
\begin{aligned}
M I & =H(A)+H(B)-H(A, B) \\
& \left.=\sum_{a, b} p_{A B}(a, b) \log \frac{p_{A B}(a, b)}{p_{A}(a) \cdot p_{B}(b)}\right) \\
N M I & =(H(A)+H(B)) / H(A, B) \\
& =\sum_{a, b} \frac{p_{A}(a) \log p_{A}(a)+p_{B}(b) \log p_{B}(b)}{p_{A B}(a, b) \log p_{A B}(a, b)}
\end{aligned}
$$

with $H$ representing the Shannon entropy.

In the present paper we restrict the geometric transformation to rigid body transformation $T_{\alpha}$ ( $T_{\alpha}$ consists of 3 translations and 3 rotations). The rigid body assumption is well satisfied inside the skull in 3-D scans of the head. The tri-linear partial volume (PV) interpolation is used to update the joint histogram for non-grid alignment [6]. In all experiments, the joint histogram size is $64 \times 64$ because the image intensities have been linearly binned into 64 grey-levels. Similar to mutual information and normalized mutual information, the registration methods based on the FDOD function and NFDOD function state that the images are geometrically aligned by the transformation $T_{\alpha^{*}}$ for which $F D O D$ or NFDOD is maximal.

The images are initially positioned such that their centers coincide. Powell's multidimensional direction set method is then used to maximize it. This method is a reasonable compromise between robustness and speed. Powell's method involves a series of one-dimensional minimizations for each dimensional; these minimizations are carried out by Brent's one-dimensional optimization. Having found an optimum in one direction, the minimization is continued in the next direction, starting from the current position. Once all six parameters ( 3 translations and 3 rotations) have been optimized, the loop is repeated until convergence is reached. In the present work, the fractional precision convergence parameters for the Brent and Powell optimization algorithm are set to $10^{-3}$ and $10^{-5}$ respectively $[16,17]$.

\section{Registration Experiments}

To demonstrate the performance of the proposed FDOD function and NFDOD function and compare them with MI and NMI, a number of experiments have been performed. There are two parts in our experiments: in the first part, synthetic MR images obtained from the Brainweb Database (available online at http://www.bic.mni.mcgill.ca/brainweb) [18] are use to perform a preliminary task; in the second part, for further investigation and comparison, we use the clinical data provided by Vanderbilt University [19].

\subsection{Registration Functions}

To visually evaluate the performance of our registration algorithms, we respectively plot the changes of the FDOD and NFDOD functions with respect to the rotation around 
an in-plane axis, and the other two measures based on MI and NMI are also considered for comparison. In this section, synthetic MR images obtained from the Brainweb Database are used to evaluate our methods. It should be noted that the images have been correctly registered, so every curve should reach its global maximum at the position zero.
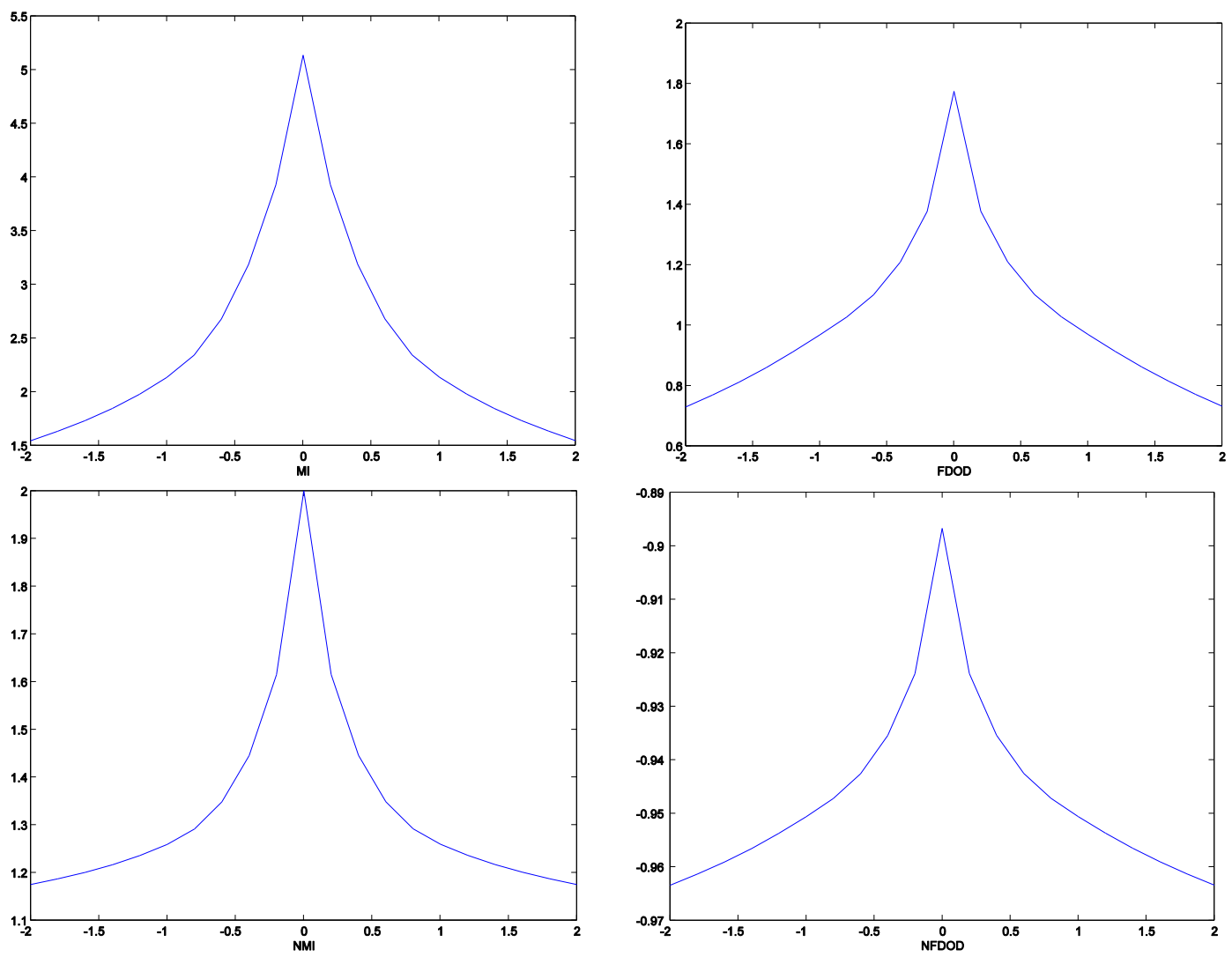

\section{Figure 1. Registration Functions for Simulated MR Images Matching. In Each Case, $x$ Axis Represents the Rotation in Degrees and $y$ axis Represents the Registration Measure}

Observing Figure 1, it is clear that the four functions have similar curves, all the curves are smooth and reach the global maximum at the position zero. The FDOD function has a range of $0 \square 2$, and the NFDOD function does not exceed the range $-1 \square 0$. On the other hand, the ranges of both mutual information and normalized mutual information are some larger. This means that given the same stopping criterion of Powell, the registration using the FDOD function and the NFDOD function may perform less iterations. We can draw the primary conclusion that the registration methods based on the FDOD function and NFDOD function respectively are feasible and competitive.

\subsection{Registration Results}

Further experiments are carried out on clinical data furnished by Vanderbilt University as the practice data set. This data set contains stereo tactically acquired MR, CT and PET images, which have been edited to remove stereo tactic markers. For an in depth description of this database, please see $[19,20]$. The image and the voxel sizes for this data set are listed in Table 2 . There is only one subject in the practice data set for each registration task and in each task, CT or PET image is taken as floating image while MR image is always the reference. The images are not preregistered other than having their centers aligned and their axes orientation corresponding. The CT image is 4-voxel 
subsampled in $x$ and $y$ directions and 2-voxel subsampled in $z$ direction to accelerate the registration process for all the registration tasks in this work. We compare our results with the stereo tactic registration solutions provided by Vanderbilt University. The difference between the reference and each of the registration solutions computed is evaluated at eight points near the brain surface. The registration error of our results is computed as follows:

$$
\text { error }=\frac{1}{8} \sum_{i=1}^{8}\left\|q_{i, \text { reference }}-q_{i}\right\|_{2}
$$

where $q_{i, \text { reference }}$ represents the $i_{\text {th }}$ point's coordinate provided by Vanderbilt University, and $q_{i}$ is the coordinate we computed. We define the diagonal distance in a voxel to indicate the size of a voxel. For CT to MR registration, the size of a voxel is the diagonal distance in a nonrectified MR image's voxel, shown at below:

$$
\sqrt{1.25^{2}+1.25^{2}+4.00^{2}} \approx 4.373(\mathrm{~mm})
$$

and for PET to MR, the size of a voxel is the diagonal distance in a voxel belonging to PET image, depicted as follows:

$$
\sqrt{2.590723^{2}+2.590723^{2}+8.00^{2}} \approx 8.799(\mathrm{~mm})
$$

If the error of a registration method is smaller than the size of a voxel, we can say this method succeed and reach the subvoxel accuracy.

Table 2. Image Sizes (in voxel) and Voxel Sizes (in $\mathrm{mm}$ ) of the Practice Data Set We Used

\begin{tabular}{|l|l|c|}
\hline Image & Image sizes in voxel & Voxel sizes in $\mathrm{mm}$ \\
\hline CT & {$[512 \times 512 \times 29]$} & {$[0.65 \times 0.65 \times 4.00]$} \\
\hline PET & {$[128 \times 128 \times 15]$} & {$[2.59 \times 2.59 \times 8.00]$} \\
\hline T1, T2, PD & {$[256 \times 256 \times 26]$} & {$[1.25 \times 1.25 \times 4.00]$} \\
\hline T1rect & {$[256 \times 256 \times 26]$} & {$[1.26 \times 1.26 \times 4.05]$} \\
\hline T2rect & {$[256 \times 256 \times 26]$} & {$[1.27 \times 1.27 \times 4.07]$} \\
\hline PDrect & {$[256 \times 256 \times 26]$} & {$[1.26 \times 1.26 \times 4.10]$} \\
\hline
\end{tabular}

To examine how robustly different initial misalignments may be recovered using the measures, one set of 50 randomized transformations are used. These are derived by perturbing the 6 rigid reference parameters with random translations and rotations of sizes $\left(30 \mathrm{~mm}, 30^{\circ}\right)$. These are then used as starting estimated for the Powell's optimization algorithm for each of the four measures. Four image pairs are considered in this experiment: CT and MR-T1, CT and MR-PD, PET and MR-T1, PET and MR-PD. Tables 3, 4 summarize the registration results, the success rate, the efficiency (measured by the mean number of the registration function evaluations), and the mean registration error, in $m m$, for all successful registrations are given. Observing Tables 3 and 4, we find that the NFDOD registration method performs best in most cases, it has a tiny superiority in both success rate and efficiency, and the four registration criteria have comparable accuracy. 
Table 3. Registration Results for CT and MR Image Pairs with Respect to Mean Error(in $\mathrm{mm}$ ), Efficiency (the Mean Number of the Registration Function Evaluations) and Success Rate

\begin{tabular}{|c|l|l|l|l|}
\hline Image pairs & Measure & Error & Efficiency & Mismatch \\
\hline \multirow{3}{*}{$\begin{array}{c}\text { CT } \\
\text { MR_T1 }\end{array}$} & MI & 1.9931 & 722.4681 & 3 \\
\cline { 2 - 5 } & FDOD & 3.4432 & 775.9787 & 3 \\
\cline { 2 - 5 } & NMI & 1.8916 & 590.9333 & 5 \\
\cline { 2 - 5 } & NFDOD & 3.1070 & 549.0652 & 4 \\
\hline CT & MI & 2.5280 & 752.1258 & 2 \\
\cline { 2 - 5 } $\mid$ & FDOD & 2.8459 & 709.0625 & 1 \\
\cline { 2 - 5 } MR_PD & NMI & 2.0351 & 2.0351 & 5 \\
\cline { 2 - 5 } & NFDOD & 2.3109 & 2.3109 & 2 \\
\hline
\end{tabular}

Table 4. Registration Results for PET and MR Image Pairs with Respect to Mean Error (in $\mathrm{mm}$ ), Efficiency (the Mean Number of The Registration Function Evaluations) and Success Rate

\begin{tabular}{|c|l|l|l|l|}
\hline Image pairs & Measure & Error & Efficiency & Mismatch \\
\hline \multirow{3}{*}{$\begin{array}{l}\text { PET } \\
\text { MR_T1 }\end{array}$} & MI & 6.3564 & 902.3043 & 27 \\
\cline { 2 - 5 } & FDOD & 6.4873 & 1016.0000 & 24 \\
\cline { 2 - 5 } & NMI & 7.0107 & 637.0800 & 0 \\
\cline { 2 - 5 } & NFDOD & 7.1242 & 590.8000 & 0 \\
\hline \multirow{2}{*}{$\begin{array}{c}\text { PET } \\
\text { MR_PD }\end{array}$} & MI & 2.5280 & 944.4138 & 21 \\
\cline { 2 - 5 } & FDOD & 2.8459 & 948.7500 & 20 \\
\cline { 2 - 5 } & NMI & 2.0351 & 857.0600 & 0 \\
\cline { 2 - 5 } & NFDOD & 2.3109 & 742.4200 & 0 \\
\hline
\end{tabular}

In order to examine the performance of the proposed methods over a larger database of images, the four measures are used to recover alignment between all the image pairs in the whole practice data. The initial registration parameter is set to zero vector $[0,0,0,0,0,0]$ which is a typical starting estimate for automated registration in clinical use. Tables 5 and 6 summarize the registration results for the four measures. The registration error, in $m m$, and the number of the registration function evaluations are given. Registration using mutual information and FDOD function perform worse which resulted in four misregistrations respectively. The normalized mutual information failed twice. Again, the normalized FDOD function provides the best overall behavior with only once failure and requires the lowest number of registration function evaluations through almost all the registration tasks.

Overall, the performance of the normalized FDOD function seemed more robust and more efficient, although the number of experiments is too small to dare make bold statements about this.

Table 5. Registration Error (in $\mathrm{mm}$ )/ Number of Registration Function Evaluations for CT and MR Image Pairs

\begin{tabular}{|l|l|l|l|l|l|l|}
\hline Method & CT-T1 & CT-T2 & CT-PD & $\begin{array}{l}\text { CT- } \\
\text { T1rect }\end{array}$ & $\begin{array}{l}\text { CT- } \\
\text { T2rect }\end{array}$ & CT-PDrect \\
\hline MI & $1.9148 / 496$ & $3.7645 / 660$ & $2.4748 / 685$ & $2.2451 / 685$ & $4.2507 / 672$ & $\mathbf{4 . 4 9 9 6 / 5 6 8}$ \\
\hline FDOD & $3.4445 / 577$ & $3.7463 / 737$ & $2.6463 / 599$ & $2.9925 / 607$ & $4.3176 / 755$ & $\mathbf{4 . 8 3 1 0 / 4 9 1}$ \\
\hline NMI & $1.9150 / 420$ & $3.7659 / 568$ & $1.5440 / 589$ & $2.2137 / 512$ & $4.2384 / 572$ & $4.3684 / 489$ \\
\hline NFDOD & $3.4797 / 427$ & $3.7359 / 505$ & $1.5604 / 424$ & $2.0204 / 435$ & $4.2964 / 564$ & $4.3242 / 420$ \\
\hline
\end{tabular}

Notes: values in bold denote those misregistrations. 
Table 6. Registration Error (in $\mathrm{mm}$ )/ Number of Registration Function Evaluations for PET and MR Image Pairs

\begin{tabular}{|l|l|l|l|l|l|l|}
\hline Method & PET-T1 & PET-T2 & PET-PD & $\begin{array}{l}\text { PET- } \\
\text { T1rect }\end{array}$ & $\begin{array}{l}\text { PET- } \\
\text { T2rect }\end{array}$ & $\begin{array}{l}\text { PET- } \\
\text { PDrect }\end{array}$ \\
\hline MI & $8.2334 / 740$ & $\mathbf{9 . 6 2 5 5 / 6 7 4}$ & $7.8427 / 823$ & $\begin{array}{l}\mathbf{9 . 2 6 4 8 / 1 0 1} \\
\mathbf{7}\end{array}$ & $8.7327 / 677$ & $\mathbf{9 . 7 7 1 6 / 8 3 2}$ \\
\hline FDOD & $5.4880 / 771$ & $\mathbf{9 . 4 8 9 0 / 6 5 2}$ & $7.8346 / 906$ & $\mathbf{9 . 1 6 7 6 / 7 7 2}$ & $\mathbf{9 . 0 9 2 9 / 4 9 3}$ & $7.4520 / 757$ \\
\hline NMI & $7.7018 / 440$ & $\mathbf{9 . 8 6 5 3 / 5 9 2}$ & $7.8991 / 666$ & $7.1251 / 420$ & $\mathbf{9 . 0 6 2 5 / 4 1 0}$ & $6.1629 / 744$ \\
\hline NFDOD & $6.4547 / 427$ & $\mathbf{9 . 6 4 1 8 / 5 1 3}$ & $7.8888 / 574$ & $7.5736 / 412$ & $8.2162 / 420$ & $6.2740 / 417$ \\
\hline
\end{tabular}

Notes: values in bold denote those misregistrations.

\section{Conclusions}

The FDOD function is a new measure of information discrepancy, and in contrast to the KLD measure it has more appealing mathematical characteristics, and has been successfully used in many fields. Motivated by these facts, we investigate the performance of this function used in medical image registration. More importantly, we define the normalized form of the FDOD function and apply it to registration problem too. Our experiments on multi-modality brain images show that both the proposed methods based on the FDOD function and NFDOD function are feasible. Comparing the behavior among mutual information, FDOD function, normalized mutual information and normalized FDOD function, the normalized FDOD function computationally more efficient without sacrificing registration accuracy. However, many good characteristics of the FDOD function have not emerged when used in the medical image registration problem. Our future work is to explore further to make the most of these good characteristics, for example, to register multiple images simultaneously.

\section{Acknowledgments}

The clinical images and their standard transformations were provided as part of the project, "Retrospective Image Registration Evaluation", National Institutes of Health, Project Number 1 R01 NS33926-01, Principal Investigator Prof. J. Michael Fitzpatrick, Vanderbilt University, Nashville, TN. This work is supported by Shandong Provincial Natural Science Foundation (ZR2013CL014), China.

\section{References}

[1] B. A. Ardekani, S. Guckemus, A. Bachman, M. J. Hoptman, M. Wojtaszek and J. Nierenberg, "Quantitative comparison of algorithms for inter-subject registration of 3D volumetric brain MRI scans", Journal of Neuroscience Methods, vol. 142, no. 1, (2005), pp. 67-76.

[2] M. Pan, J. Jiang, F. Zhang and Q. Rong, "Medical image registration using Fourier-mellin transform", Journal of Computing Information Systems, vol. 11, no. 3, (2015), pp. 1141-1148.

[3] H. Luo, "Study on mutual information medical image registration based on an algorithm", International Journal of Hybrid Information Technology, vol. 8, no.9, (2015), pp. 353-360.

[4] S. Luo and X. Li, "Implementation of mutual information based multi-modality medical image registration", Proceedings of the 22th Annual EMBS International Conference, (2000), pp. 1447-1450.

[5] C. Studholme, D. L. G. Hill and D. J. Hawkes, "An overlop invariant entropy measures of 3D medical image alignment”, Pattern Recognition, vol. 32, no. 1, (1999), pp. 71-86.

[6] F. Maes, A. Collignon, D. Vandermeulen, G. Marchal and P. Suetens, "Multimodality image registration by maximization of mutual information", IEEE Transactions on Medical Imaging, vol. 16, no. 2, (1997), pp. 187-198.

[7] B. Li, G. Yang, J. L. Coatrieux, B. Li and H. Shu, "3D nonrigid medical image registration using a new information theoretic measure", Physics in Medicine \& Biology, vol. 60, no. 22, 21 (2015), pp. 87678790.

[8] F. P. Oliveira and J. M. Tavares, "Medical image registration: a review", Computer Methods in Biomechanics and Biomedical Engineering, vol. 17, no. 2, (2014), pp.73-93. 
[9] W Zhou, L. Zhang, Y. Xie and C. Liang, "A novel technique for prealignment in multimodality medical image registration”, BioMed Research International, vol. 2014, no. 4, (2014), pp. 726852-726852.

[10] S. Damas, O. Cordon and J. Santamaria, "Medical image registration using evolutionary computation: an experimental survey", Computational Intelligence Magazine, vol. 6, no. 2, (2011), pp.26-42.

[11] W.W. Fang, "The characterization of a measure of information discrepancy", Information Sciences, vol. 125, no. 1-4, (2000), pp.207-232.

[12] W.W. Fang, F. S. Roberts and Z. R. Ma, “A measure of discrepancy of multiple sequences”, Information Sciences, vol. 137, no. 1-4, (2001), pp.75-102.

[13] W.W. Fang, "On a global optimization problem in the study of information discrepancy", Center for Discrete Mathematics \& Theoretical Computer Science, vol. 1, no. 4, (2012), pp.387-408.

[14] J. H. Wang, W.W. Fang, L. J. Liang and R. S. Chen, "Gene's functional arrangement as a measure of the phylogenetic relationships of microorganisms", Journal of Biological Physics, vol. 28, no.1, (2002), pp.55-62.

[15] L. X. Jin, W. W. Fang and H. W. Tang, "Prediction of protein structural classes by a new measure of information discrepancy", Computational Biology and Chemistry, vol. 27, no. 3, (2003), pp.373-380.

[16] W. H. Press, B. P. Flannery, S. A. Teukolsky and W. T. Vettering, "Numerical Recipes in C", Cambridge University Press, Cambridge, (1992).

[17] J. P. W. Pluim, J. B. A. Maintz, and M. A.Viergever, "Mutual information matching in multiresolution contexts". Image and Vision Computing, vol. 1, no. 1-2, (2001), pp. 45-52.

[18] D. L. Collins, A. P. Zijdenbos, V. Kollokian, J. G. Sled, N. L. Kabani, C. J. Holmes and A. C. Evans, "Design and construction of a realistic digital brain phantom", IEEE Transactions on Medical Imaging, vol. 17, no. 3, (1998), pp.463-468.

[19] Vanderbilt Univ., Nashville, TN. Retrospective Image Registration Evaluation (RIRE). Available on line at: http://www.insight-journal.org/rire/.

[20] J. West, J. M. Fitzpatrick and Y. W. Matthew, "Comparison and evaluation of retrospective intermodality brain image registration techniques", Journal of Computer Assisted Tomography, vol. 21, no. 4 , (1997), pp.554-66.

\section{Authors}

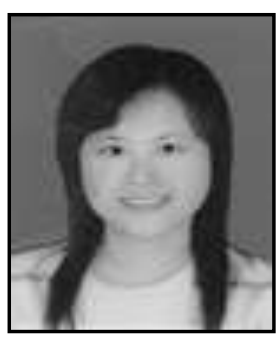

Shaoyan Sun, she is a lecturer of School of Mathematics and Statistics Science, Ludong University, Yantai, China. Her research interests are medical image processing and systems biology.

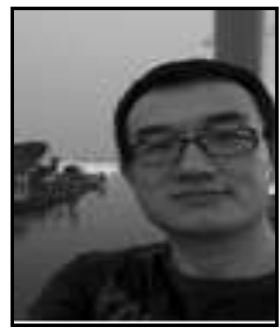

Fengnan Sun, he is a doctor of Yantaishan Hospital, Yantai, china. His research direction is medical image analysis. 
International Journal of Hybrid Information Technology

Vol. 10, No.11 (2017) 